\title{
基于苯乙烯和苯并噻二唑共聚场效应发光高分子材料的 设计合成及性能研究
}

\author{
刘情情 $a, b$ 张逸寒 $a, b$ 高灿 ${ }^{a}$ 王天禹 ${ }^{a}$ 胡文平 ${ }^{a, c}$ 董焕丽 ${ }^{*}, a, b$ \\ $\left({ }^{a}\right.$ 中国科学院化学研究所 北京分子科学国家重点实验室 有机固体重点实验室 北京 100190) \\ ${ }^{b}$ 中国科学院大学 北京 100049) \\ ( ${ }^{c}$ 天津大学理学院 天津市分子光电科学重点实验室 天津 300072)
}

\begin{abstract}
摘要 共轭高分子材料由于其优异的光电性能和可溶液加工等特性在有机光电器件中具有重要应用. 本工作采用 Stille 偶联和 Suzuki 聚合反应, 合成了两个由经典发光基元苯乙烯片段和共轭吸电子结构基元苯并噻二唑共聚的高分子材料 聚(1,2-双(2,5-双(异辛氧基)亚苯基亚乙烯基-2,1,3-苯并噻二唑)) (PVBT)和聚(1,2-双(2,5-双(正辛氧基)亚苯基亚乙烯基2,1,3-苯并噻二唑)) (nPVBT). 通过凝胶渗透色谱(GPC)、元素分析及差式扫描量热法(DSC)对 PVBT 和 nPVBT 两种高 分子材料的结构及热稳定性进行表征, 结果表明它们均具有良好的热稳定性, 分解温度约 $380{ }^{\circ} \mathrm{C}$. 由于烷氧基链的存 在，两个材料具有良好的溶解性及成膜加工性. PVBT 和 nPVBT 均表现出优异的发光特性，最大发射波长在 590 605 $\mathrm{nm}$ 范围, 溶液下苂光量子产率为 $23 \% \sim 35 \%$, 固态薄膜下量子产率为 $12 \% \sim 20 \%$. 以这两个高分子材料薄膜作为活性 层, 所制备的顶栅-底接触型有机场效应晶体管器件显示出典型的 $\mathrm{p}$ 型电荷传输性能, 空穴迁移率可达 $1.1 \times 10^{-4} \mathrm{~cm}^{2}$. $\mathrm{V}^{-1} \cdot \mathrm{s}^{-1}$, 开关比为 $10^{3} \sim 10^{4}$. 本研究为发展高性能光电集成高分子材料提供了新思路, 有望推动有机光电集成器件的 研究.

关键词＼cjkstart苯乙烯; 苯并噻二唑; 高发光; 场效应性能
\end{abstract}

\section{Synthesis and Property Study of Field-effect Emissive Conjugated Polymers Based on Styrene and Benzothiadiazole}

\author{
Liu, Qingqing ${ }^{a, b} \quad$ Zhang, Yihan $^{a, b} \quad{\text { Gao, } \text { Can }^{a}}^{a}$ Wang, Tianyu $^{a} \quad \mathrm{Hu}$, Wenping ${ }^{a, c}$ \\ Dong, Huanli*,a,b \\ ( ${ }^{a}$ Beijing National Laboratory for Molecular Sciences, Key Laboratory of Organic Solids Institute of Chemistry, \\ Chinese Academy of Sciences, Beijing 100190, China) \\ ( ${ }^{b}$ University of Chinese Academy of Sciences, Beijing 100049, China) \\ ( ${ }^{c}$ Tianjin Key Laboratory of Molecular Optoelectronic Sciences, School of Science, \\ Tianjin University, Tianjin 300072, China)
}

\begin{abstract}
Conjugated polymer materials with good photoelectric performance, solution processing ability and flexibility are widely used as active layers in optoelectronic devices. Here, using Stille and Suzuki coupling reactions, we designed and synthesized two new conjugated polymers, poly(1,2-bis(2,5-bis(iso-octyloxy)phenylenevinylene-2,1,3-benzothiadiazole)) (PVBT) and poly(1,2-bis(2,5-bis(n-octyloxy)phenylenevinylene-2,1,3-benzothiadiazole)) (nPVBT), which contain structural element styrene fragments and an conjugated unit benzothiadiazole. Styrene fragments are conducive to luminescent properties of materials, such as phenylenevinylene (PPV) derivatives, while benzothiadiazole unit is electron withdrawing, and matches with many structural units of a donor. The conjugated polymers were characterized by gel permeation chromatography (GPC), elemental analysis and differential scanning calorimetry (DSC). The results indicate that each of these two polymers has good thermal stability. Their melting points were around $240 \sim 250{ }^{\circ} \mathrm{C}$ and decomposition temperatures around $380{ }^{\circ} \mathrm{C}$. Due to the presence of the structural alkoxy chains, these two polymers exhibit good solubility, which is conducive to solution-processed film formation. PVBT and nPVBT have strong fluorescence characters with maximum emission in the range of $590 \sim 605 \mathrm{~nm}$. The photoluminescence quantum yield of these two polymers in dichloromethane solution $\left(1 \times 10^{-5}\right.$ $\mathrm{mol} \cdot \mathrm{L}^{-1}$ ) is $23 \% \sim 35 \%$, and $12 \% \sim 20 \%$ in solid films, which are annealed at $180{ }^{\circ} \mathrm{C}$ for $10 \mathrm{~min}$. Due to benzothiadiazole's regulation of molecular energy levels, the highest occupied molecular orbital (HOMO) energy level of PVBT and nPVBT
\end{abstract}

* E-mail: dh1522@iccas.ac.cn

Received May 16, 2020; published June 24, 2020.

Supporting information for this article is available free of charge via the Internet at http://sioc-journal.cn.

Project supported by the National Key Research and Development Project (Nos. 2017YFA0204503, 2018YFA0703200), the National Natural Science Foundation of China (Nos. 61890943, 51725304) and Beijing National Laboratory for Molecular Sciences (BNLMS-CXXM-202012).

项目受国家重点研发计划(Nos. 2017YFA0204503, 2018YFA0703200)、国家自然科学基金(Nos. 61890943, 51725304)和北京市分子科学中心项目(No. BNLMS- CXXM-202012)资助。 
were modulated to be -5.73 and $-5.61 \mathrm{eV}$, and the lowest unoccupied molecular orbital (LUMO) energy level were -3.37 and $-3.32 \mathrm{eV}$, respectively. Typical p-type transporting property was determined by using PVBT and nPVBT films as active layers in organic field effect transistors. Because of the improved conjugation of the skeleton structures and the close packing between benzothiadiazole of main chains, these two conjugated polymers both exhibit efficient charge transport characteristics with saturation hole carrier mobility is up to $1.1 \times 10^{-4} \mathrm{~cm}^{2} \cdot \mathrm{V}^{-1} \cdot \mathrm{s}^{-1}$ and high switching on/off ratio of $10^{3} \sim$ $10^{4}$. This work provides new insight into the development of high-performance optoelectronic conjugated polymer materials and sheds light on the research of organic optoelectronic integrated devices.

Keywords styrene; benzothiadiazole; strong emission; field-effect transporting property

\section{1 引言}

共轭高分子材料因具有优异的光电性能、溶液加工 特性及柔韧性，作为活性层被广泛应用于有机场效应晶 体管(OFET)、有机发光二极管(OLED)、有机太阳能电 池 $(\mathrm{OPV})$ 等光电子器件. 经过过去几十年的不断研究, 这些器件的性能得到了显著提高, 在很多领域显示了重 要应用 ${ }^{[1-24]}$. 相比较而言, 有机发光场效应晶体管 (OLET)作为一种兼具有机发光二极管和场效应晶体管 的小型化光电集成器件, 其发展一直严重滞后 ${ }^{[25-29]}$. 限 制 OLET 器件发展的一个主要原因是核心有机活性层材 料的严重缺乏. OLET 的活性层材料需要同时具备高光 致发光量子产率 $(\Phi)$ 和高载流子迁移率 $(\mu)$, 但二者互相 制衡, 不易兼得 ${ }^{[30]}$. 高迁移率性能要求材料具有较大的 分子共轭结构和紧密的分子链堆积, 相邻分子间电子耦 合强, 这往往导致材料苂光猝灭严重, 甚至不发光, 而 强荧光材料分子往往分子间距大, 分子间耦合作用弱, 不利于电荷的有效传输 ${ }^{[20,31]}$. 令人鼓舞的是, 最近高性 能场效应发光小分子半导体材料取得了重要进展, 系列 性能优异的小分子光电集成材料被成功设计合成出来, 为 OLET 器件研究奠定了良好的材料基础 ${ }^{[2-35]}$. 但是目 前具有优异可溶液加工性和机械性能的场效应发光共 轭高分子材料还很少, 其设计仍面临巨大挑战 ${ }^{[2,29]}$. 受 到目前高迁移率发光小分子半导体材料研究的启发, 同 时基于强发光和高迁移率共轭高分子材料的前期研究 基础 ${ }^{[1-17]}$, 我们提出了基于优异发光基元和传输基元共 聚制备光电集成共轭高分子材料的设计思想(图 1), 希 望通过协同调控基元间的连接方式和侧链工程, 譬如利 用大取代基增大发光基元间分子间距，抑制苂光严重猝 灭, 通过分子共轭骨架和分子间作用协同调控链内和链 间电荷传输, 实现发光和场效应性能的有效集成, 获得 具有场效应性能的发光共轭高分子材料新体系.

苯乙烯是一种广泛应用于发光材料的典型结构基 元, 基于苯乙烯基元的聚苯乙烯撑(PPV)及其衍生物具 有高的光致发光, 是一类重要的共轭高分子光电材料, 被广泛应用于 OLED 等电致发光器件的研究 ${ }^{[36]}$. PPV 作 为第一代共轭高分子发光材料, 被用于制备了第一个聚 合物发光二极管器件 ${ }^{[37]}$, 第一个体相异质结太阳能电 池 ${ }^{[38]}$ 和第一个有机光洜浦固体激光器 ${ }^{[39]}$. 最初对于 PPV 类材料器件的研究是通过旋涂可溶的硫盐前聚体

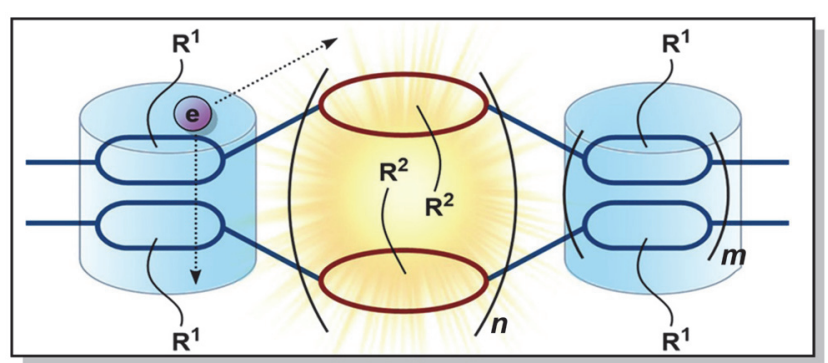

图 1 高性能场效应发光共轭高分子材料的设计思想

Figure 1 Molecular design concept for high-performance optoelectronic conjugated polymers

再高温退火的方式制备薄膜, 不具有可溶液加工特性, 限制了其器件应用 ${ }^{[37]}$. 为了提高该类材料的溶解性, 后 续研究中通常会在苯乙烯结构基元上引入合适的柔性 侧链, 这一措施一方面可有效改善材料的溶解性和成膜 性，同时由于侧链对于分子链间距离及作用力的调控， 有效抑制了因链间激子转移造成的荧光猝灭现象, 大大 提高了材料的发光特性. 譬如, 含不对称癸氧基的聚(2甲氧基-5-(3,7-二甲基辛氧基)-对亚苯基亚乙烯基) $\left(\mathrm{OC}_{1} \mathrm{C}_{10}-\mathrm{PPV}\right)$ 的固态苂光量子产率为 $9 \% \sim 10 \%{ }^{[40]}$, 含 不同烷氧基链混聚 Super Yellow 材料的荧光量子效率达 $60 \%{ }^{[41]}$, 而含烷氧基苯基侧链的聚(2-(2',5'-双(2"-乙基己 氧基)苯基)-1,4-亚苯基亚乙烯基) (BEHP-PPV)的固态量 子产率则可高达 $62 \%{ }^{[42]}$, 这些材料作为经典的共轭高分 子发光材料在 OLED 器件中已被广泛研究. 但是由于苯 乙烯共轭骨架结构体系较小, 分子结构存在构象扭曲, 不利于分子链间形成强的 $\pi-\pi$ 堆积作用, 导致大部分材 料的电荷传输性能比较差. 目前给受体(D-A 型)共轭高 分子材料由于在分子能级结构、分子内/分子间作用力以 及堆积模式和结晶行为等方面优异的调控能力而备受 关注. 其中, 苯并噻二唑(BT)结构是一种具有合适的吸 电子能力, 可与很多给体结构基元进行匹配的重要共轭 结构基元，在构建高迁移率共轭高分子材料方面显示了 重要应用 ${ }^{[2,7,12]}$. 此外, 基于 BT 和芳香类(例如苯乙烯基 元)化合物偶联衍生的高分子材料往往具有较低的最高 占据分子轨道(HOMO), 不易氧化, 所获得材料往往具 有优异的稳定特性 ${ }^{[43-45]}$. 譬如, 目前基于 $\mathrm{BT}$ 与发光基 团 9,9-二辛基荡交替共聚得到的聚( 9 ,9-二辛基荡苯并噻 二唑) (F8BT) 是一类重要的高分子光电材料, 在 OLED 器件和 OLET 器件中得到了很好的研究 ${ }^{[46]}$. 基于前面提 
出的光电分子设计思想和目前文献的相关研究工作, 我 们设计发展了一类以含辛氧基侧链的苯乙烯单元和 $\mathrm{BT}$ 偶联共聚的新型 D-A 型共轭高分子场效应发光材料, 希 望通过烷氧基侧链调控苯乙烯发光基元的间距，防止相 邻链苯乙烯共轭部分形成紧密堆积, 抑制苂光猝灭; 同 时利用 BT 的吸电子能力调控分子共轭骨架的共轭性和 链间的距离, 保证有效的链间和链内的电荷传输特性 (图式 1). 经 Stille 偶联和 Suzuki 聚合我们得到了结构单 元明确的以支链取代的共轭高分子材料聚(1,2-双(2,5双 (异辛氧基) 亚苯基亚乙烯基-2,1,3-苯并噻二唑)) (PVBT) 和以直链取代的共轭高分子材料聚(1,2-双(2,5双 (正辛氧基)亚苯基亚乙烯基)-2,1,3-苯并噻二唑)) (nPVBT). 研究结果表明, PVBT 和 nPVBT 均展现了优 异的发光特性, 固态薄膜下的荧光量子产率可高达 $20 \%$, 同时兼具典型的 $\mathrm{p}$ 型电荷传输特性, 其饱和载流 子迁移率为 $1.1 \times 10^{-4} \mathrm{~cm}^{2} \cdot \mathrm{V}^{-1} \cdot \mathrm{s}^{-1}$, 进一步结合其良好 的可溶液加和热稳定性, 预示它们在有机光电集成器件 的潜在应用.

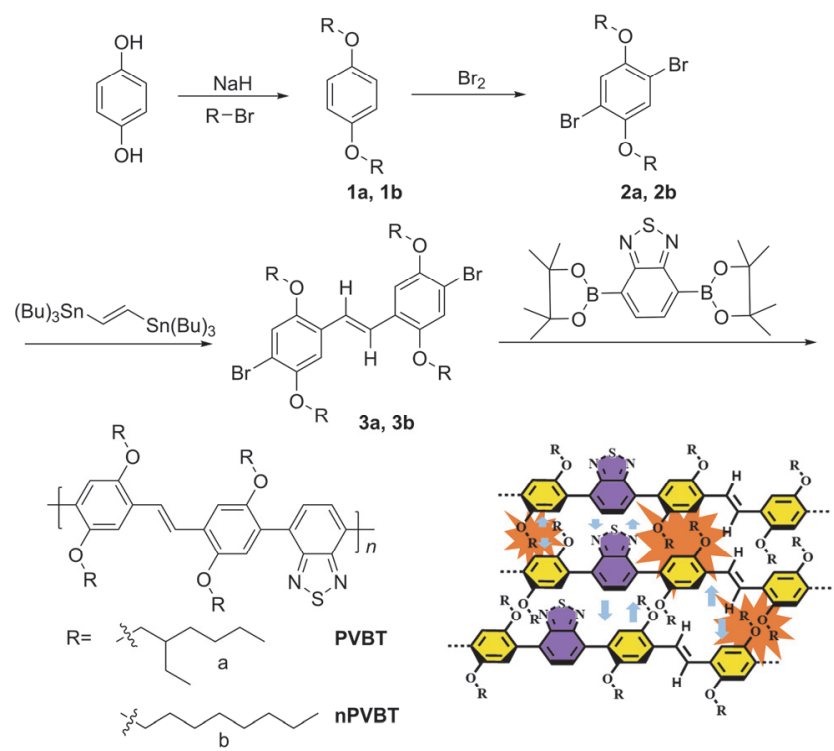

图式 1 PVBT 和 nPVBT 的合成路线及分子堆积模型

Scheme 1 Synthesis routes and packing model of PVBT and nPVBT

\section{2 结果与讨论}

\section{1 共轭高分子材料的合成及表征}

如图式 1 所示, 1,4-二辛氧基苯 $(1 \mathbf{a}, 1 \mathbf{b})$ 的合成首先在 $\mathrm{NaH}$ 的强碱条件下发生烷基化反应, 产率和后续尝试在 $\mathrm{KOH}$ 条件下反应产率基本不变. (E)-1,2-双(4-溴-2,5-双 (辛氧基)苯基)乙烯 $(3 \mathrm{a}, 3 \mathrm{~b})$ 的合成则需要底物 $(2 \mathrm{a}, 2 \mathrm{~b})$ 过 量较多, 以防止进行过多缩聚反应 ${ }^{[10]}$, 相关化合物的核 磁和单体质谱图谱见电子支持信息图 $\mathrm{S} 1 \sim \mathrm{S} 10$. 聚合反 应选择的是反应速度可控的 Suzuki 偶联反应得到的 PVBT 和 nPVBT. 中间体以及目标分子的结构经核磁共 振 ${ }^{1} \mathrm{H} N M R 、{ }^{13} \mathrm{C} N M R$ 、质谱 HRMS 及 GPC 验证分子
结构, 测定分子量分布的图谱见 S11, S12, PVBT 的数均 分子量 $\left(M_{\mathrm{n}}\right)$ 为 $32.5 \mathrm{kDa}$, 多分散指数 $(\mathrm{PDI})$ 为 2.38 , 与 nPVBT $\left(M_{\mathrm{n}}=16.6 \mathrm{kDa}, \mathrm{PDI}=1.65\right)$ 相比有一定差异, 这 可能是由于烷氧基链的不同会影响高分子材料的溶解 性, 从而影响后续索式提取过程 ${ }^{[47]}$.

\section{2 高分子材料的热学性}

如图 2(A) 所示, PVBT 和 nPVBT 均具有较好的热稳 定性, 热分解温度 $\left(T_{\mathrm{d}}\right.$, 对应 $5 \%$ 的重量损失)均在 $380{ }^{\circ} \mathrm{C}$ 左右. 第 2 次热循环的 DSC 曲线 [图 2(B)]显示两个高分 子材料在 $240 \sim 255{ }^{\circ} \mathrm{C}$ 出现吸热峰, 对应共轭高分子材 料的熔点 $\left(T_{\mathrm{m}}\right)$. 进一步升温, PVBT 至 $300{ }^{\circ} \mathrm{C}, \mathrm{nPVBT}$ 至 $350{ }^{\circ} \mathrm{C}$, 均没有发现相转变过程, 说明聚合物分解温度 前只有熔融点, 没有其他相转变.
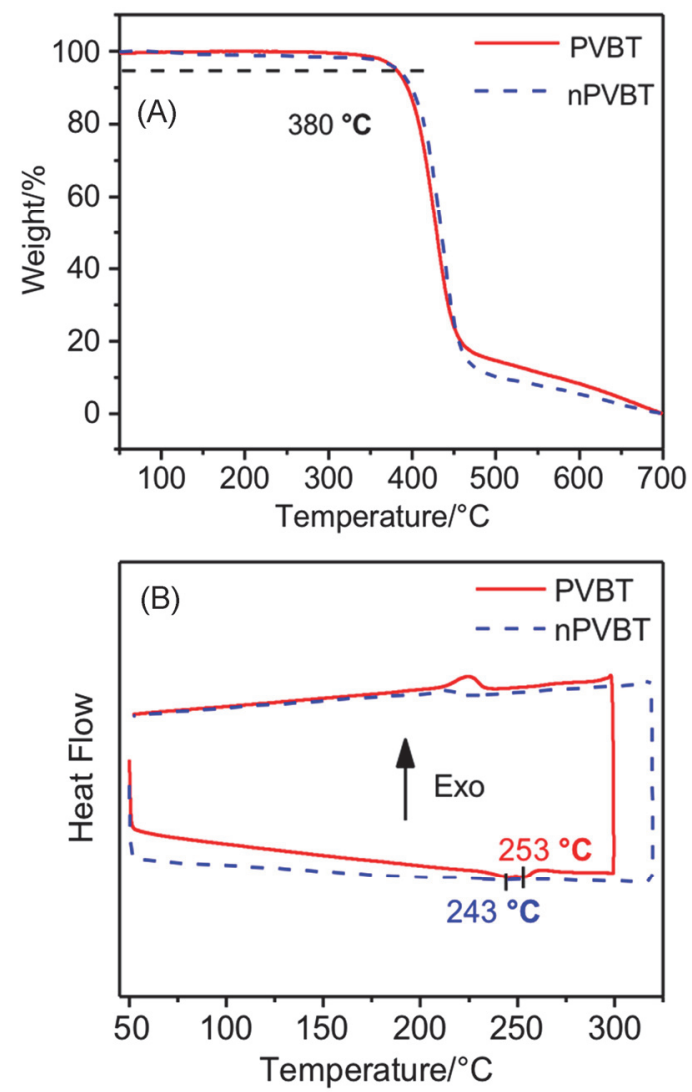

图 2 材料的(A)热重分析 TGA 和(B)二次扫描 DSC 热分析图(氮气气 氛下以 $10 \mathrm{~K} \cdot \mathrm{min}^{-1}$ 的速率加热或冷却)

Figure 2 (A) TGA and (B) second-scan DSC thermograms of the polymers (Under nitrogen atmosphere with a heating or cooling rate of 10 $\mathrm{K} \cdot \mathrm{min}^{-1}$ )

\section{3 光物理性质和光电效应}

光物理性能测试结果如图 3 所示, 室温下 PVBT 和 $\mathrm{nPVBT}$ 的溶液和薄膜均具有较高的光致发光特性, 在 $\mathrm{CH}_{2} \mathrm{Cl}_{2}$ 稀溶液中其最大吸收波长处的摩尔吸光系数分 别可达 30000 和 $32000 \mathrm{~L} \cdot \mathrm{mol}^{-1} \cdot \mathrm{cm}^{-1}$, 说明材料具有较 好的吸光能力, 并且在 $365 \mathrm{~nm}$ 紫外灯照射下发出亮黄 
(A)
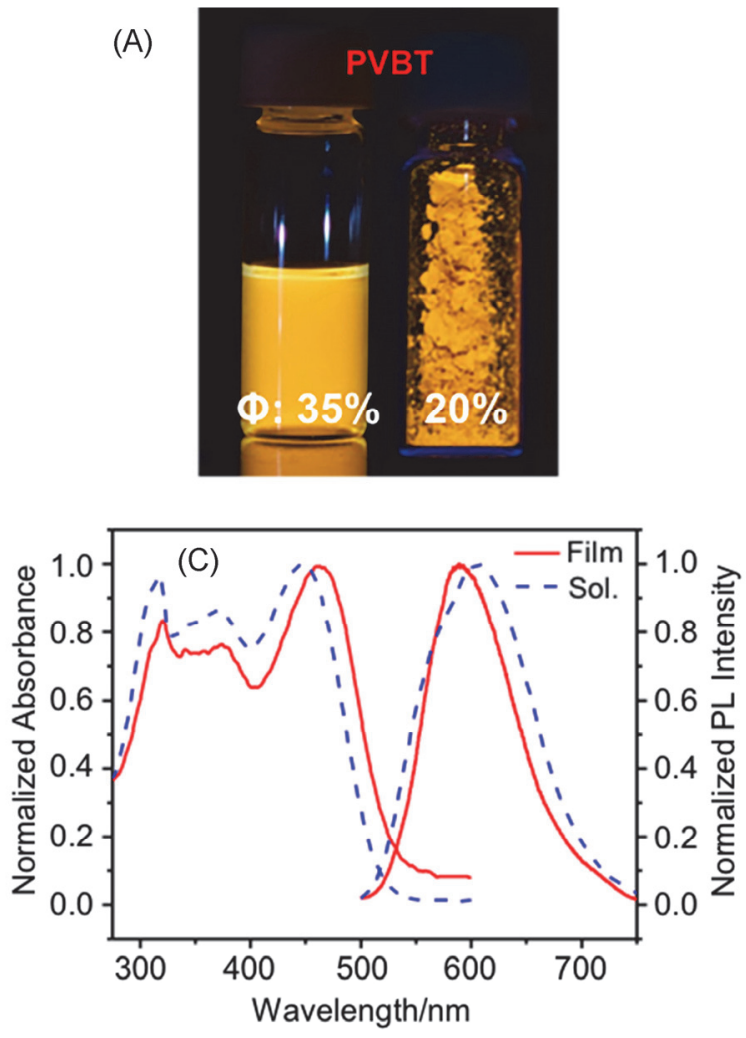

(B)
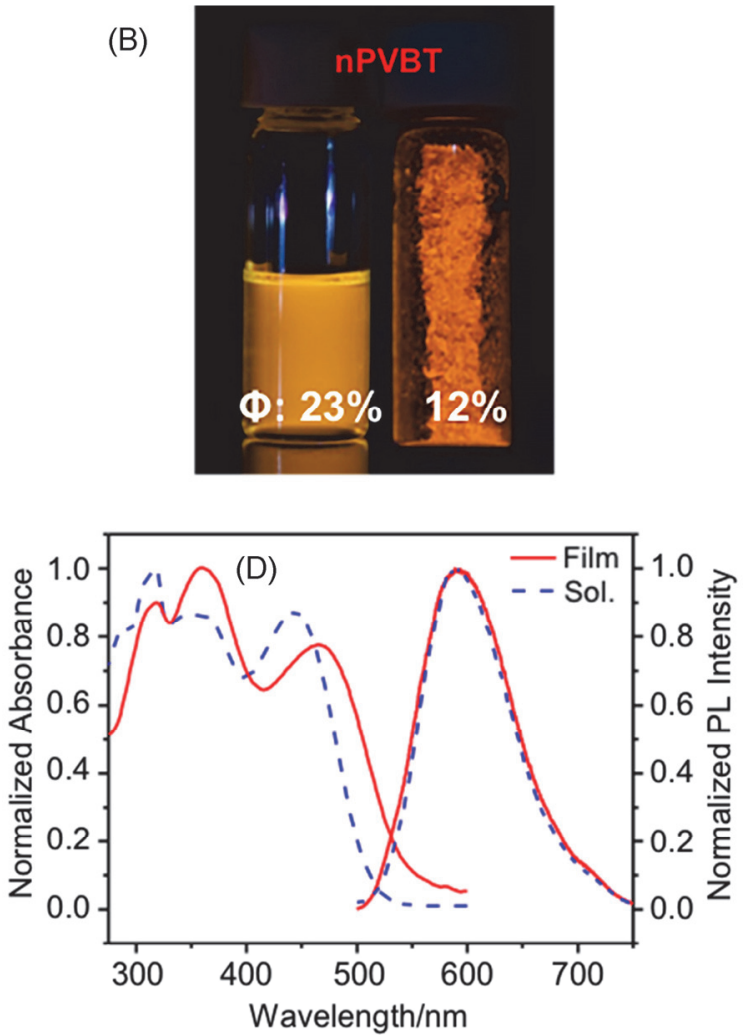

图 3 (A, B) $365 \mathrm{~nm}$ 光照下的苂光照片及 PVBT (C) 和 $\mathrm{nPVBT}$ (D)的 UV-vis/PL 光谱(实线: 薄膜在 $180{ }^{\circ} \mathrm{C}$ 下退火 $10 \mathrm{~min}$; 虚线: 材料溶于 $\mathrm{CH}_{2} \mathrm{Cl}_{2}$ 的稀溶液浓度为 $\left.1 \times 10^{-5} \mathrm{~mol} \cdot \mathrm{L}^{-1}\right)$

Figure 3 (A, B) Fluorescence photographs under UV (365 nm) illumination. Normalized UV-vis absorption/PL spectra of PVBT (C) and nPVBT (D) (Solid lines: films annealed at $180{ }^{\circ} \mathrm{C}$ for $10 \mathrm{~min}$; Dash lines: polymers in dichloromethane $\left(1 \times 10^{-5} \mathrm{~mol} \cdot \mathrm{L}^{-1}\right)$

色的光[图 3(A, B)]. 图 3(C) 和 3(D) 分别给出了两个高分 子材料在 $\mathrm{CH}_{2} \mathrm{Cl}_{2}$ 稀溶液 $\left(1 \times 10^{-5} \mathrm{~mol} \cdot \mathrm{L}^{-1}\right)$ 和在 $180{ }^{\circ} \mathrm{C}$ 下退火 $10 \mathrm{~min}$ 的薄膜状态下的归一化紫外-可见吸收光 谱及苂光光谱. 从紫外-可见吸收光谱可以看出: 两个 高分子材料薄膜均在 $460 \mathrm{~nm}$ 附近出现明显吸收峰, 这 归因于共轭高分子主链的 $\pi-\pi *$ 跃迁. PVBT 和 nPVBT 薄 膜显示出相似的紫外-可见吸收光谱, 其光学带隙约为 $2.30 \mathrm{eV}$, 这表明烷氧基链对芳香核的光物理性质影响较 小(表 1) ${ }^{[47]}$. PVBT 和 nPVBT 的薄膜吸收光谱相对其稀 溶液中的吸收光谱均具有相似的红移, 这表明由于部分 高分子链之间的强烈分子间相互作用, 在薄膜中形成一 定的聚集结构. 苂光光谱显示在薄膜和稀溶液下, PVBT 和 nPVBT 的最大发射光谱在 $590 \sim 605 \mathrm{~nm}$ 之间, 相比 于同样含有辛氧基取代的聚(2-甲氧基-5-(2'-乙基已氧 基)-1,4-亚苯基亚乙烯基) (MEH-PPV)最大发射波长在 $580 \mathrm{~nm}$ 左右, BT 的引入增强了分子间相互作用和分子 骨架结构的共轭性, 引起其光谱发生了明显的红移 ${ }^{[29]}$, 这对于提升其电荷传输性能具有非常重要的意义. 测试 所得的两个高分子材料在 $\mathrm{CH}_{2} \mathrm{Cl}_{2}$ 溶液和薄膜中的光致 发光苂光量子产率的相关数据汇总于表 1. 很明显, PVBT 和 nPVBT 在 $\mathrm{CH}_{2} \mathrm{Cl}_{2}$ 中均具有高的苂光量子产率 $(\Phi)$, 可达 $20 \% \sim 35 \%$, 即使在固态薄膜状态下, 两个材
料仍具有较好的荧光特性，其荧光量子产率在 $12 \%$ $20 \%$ 之间. 固态条件下烷氧基链由支链变为直链时, 相 应的苂光量子产率有所下降, 这主要是由于相比于支链 分子，基于直链的分子链间更容易获得规整和紧密的堆 积结构, 这也说明侧链工程在调控共轭高分子材料堆积 结构和相应光电性能方面的重要性 ${ }^{[48]}$. 同时, 对比 PVBT 和 nPVBT 的光谱可以发现二者具有较大的 Stokes 位移, 并且二者薄膜的 Stokes 位移 $(131 \mathrm{~nm}, 127 \mathrm{~nm})$ 与 稀溶液(157 nm, $146 \mathrm{~nm}$ )相似, 吸收与发射光谱的重叠 面积也较小, 预示其较大的激发态驰豫特性和较低的自 吸收, 这对于后续高效器件的构筑和激光的实现具有重 要作用 ${ }^{[33]}$. 此外, 我们测试了 PVBT 和 nPVBT 的时间分 辨苂光光谱(图 S13), 相关数据汇总于表 2. 实验结果表 明, PVBT 和 nPVBT 在稀溶液中的苂光强度呈单指数衰 减, 二者的苂光寿命分别为 $2.85 \mathrm{~ns}$ 和 $3.07 \mathrm{~ns}$, 也说明了 分子在稀溶液中以单个分子形式存在，只有一个激发 态. 而薄膜状态下的 PVBT 和 nPVBT 的荧光强度呈双 指数衰减, 二者的平均苂光寿命分别为 $1.32 \mathrm{~ns}$ 和 1.60 ns (双指数衰减模型拟合见表 S1), 此结果表明薄膜样品 中存在两种不同的激发态形式, 这可能是由于分子不同 的聚集状态产生的. 由辐射跃迁速率公式 $k_{\mathrm{r}}=\Phi / \tau$ 和非 辐射跃迁速率公式 $k_{\mathrm{nr}}=1 / \tau-k_{\mathrm{r}}$ 可得, PVBT 在溶液和薄 
表 1 PVBT 和 nPVBT 的光物理性能

Table 1 Optical physical properties of PVBT and nPVBT

\begin{tabular}{|c|c|c|c|c|c|c|c|c|c|}
\hline \multirow{2}{*}{ Sample } & \multicolumn{2}{|c|}{$\lambda_{\text {max, abs }} / \mathrm{nm}$} & \multicolumn{2}{|c|}{$\lambda_{\max , \mathrm{PL}} / \mathrm{nm}$} & \multicolumn{2}{|c|}{$\Phi / \%$} & \multirow{2}{*}{$\Delta E_{\mathrm{g}}^{\mathrm{opt} c} / \mathrm{eV}$} & \multirow{2}{*}{$\mathrm{HOMO}^{d} / \mathrm{eV}$} & \multirow{2}{*}{ LUMO/eV } \\
\hline & Sol $^{a}$ & Film $^{b}$ & $\mathrm{Sol}^{a}$ & Film $^{b}$ & $\mathrm{Sol}^{a}$ & Film $^{b}$ & & & \\
\hline PVBT & 448 & 460 & 605 & 591 & 35 & 20 & 2.36 & -5.73 & -3.37 \\
\hline nPVBT & 443 & 466 & 589 & 593 & 23 & 12 & 2.29 & -5.61 & -3.32 \\
\hline
\end{tabular}

${ }^{a}$ Polymers in dichloromethane $\left(1 \times 10^{-5} \mathrm{~mol} \cdot \mathrm{L}^{-1}\right) ;{ }^{b}$ Film were annealed at $180{ }^{\circ} \mathrm{C}$ for $10 \mathrm{~min} ;{ }^{c}$ Calculated from the film absorption onsets according to $\Delta E_{\mathrm{g}}$ opt $=$ $1240 / \lambda_{\text {onset; }}{ }^{d}$ HOMO levels were obtained by ultraviolet photoelectron spectroscopy.

表 2 PVBT 和 nPVBT 的时间分辨苂光光谱汇总

Table 2 A summery of time-resolved PL measurements of PVBT and nPVBT

\begin{tabular}{|c|c|c|c|c|c|c|}
\hline \multirow{2}{*}{ Sample } & \multicolumn{2}{|c|}{$\tau^{a} / \mathrm{ns}$} & \multicolumn{2}{|c|}{$k_{\mathrm{r}}^{b} / \mathrm{s}^{-1}$} & \multicolumn{2}{|c|}{$k_{\mathrm{nr}}^{c} / \mathrm{s}^{-1}$} \\
\hline & Sol & Film & Sol & Film & Sol & Film \\
\hline PVBT & 2.85 & 1.32 & $1.2 \times 10^{8}$ & $1.5 \times 10^{8}$ & $2.3 \times 10^{8}$ & $6.1 \times 10^{8}$ \\
\hline nPVBT & 3.07 & 1.60 & $7.5 \times 10^{7}$ & $7.5 \times 10^{7}$ & $2.6 \times 10^{8}$ & $5.5 \times 10^{8}$ \\
\hline
\end{tabular}

${ }^{a}$ Fluorescence lifetime; ${ }^{b}$ Radiative deactivation rate calculated according to $k_{\mathrm{r}}=\Phi / \tau ;{ }^{c}$ Non-radiative deactivation rate calculated according to $k_{\mathrm{nr}}=1 / \tau-k_{\mathrm{r}}$ (Sol: Polymers in dichloromethane $\left(1 \times 10^{-5} \mathrm{~mol} \cdot \mathrm{L}^{-1}\right)$; Film: Annealed at $180{ }^{\circ} \mathrm{C}$ for $\left.10 \mathrm{~min}\right)$.

膜中的辐射跃迁速率 $\left(k_{\mathrm{r}}\right)$ 分别为 $1.2 \times 10^{8} \mathrm{~s}^{-1}$ 和 $1.5 \times 10^{8}$ $\mathrm{s}^{-1}$, 非辐射跃迁速率 $\left(k_{\mathrm{nr}}\right)$ 分别为 $2.3 \times 10^{8} \mathrm{~s}^{-1}$ 和 $6.1 \times 10^{8}$ $\mathrm{s}^{-1}$; nPVBT 在溶液和薄膜中的辐射跃迁速率 $\left(k_{\mathrm{r}}\right)$ 分别为 $7.5 \times 10^{7} \mathrm{~s}^{-1}$ 和 $7.5 \times 10^{7} \mathrm{~s}^{-1}$, 非辐射跃迁速率 $\left(k_{\mathrm{nr}}\right)$ 分别为 $2.6 \times 10^{8} \mathrm{~s}^{-1}$ 和 $5.5 \times 10^{8} \mathrm{~s}^{-1}$, 可以看出, 两个分子的薄膜 样品中非辐射跃迁速率较其溶液中都明显增大, 表明薄 膜状态使激子猝灭的缺陷增加, 从而使材料的苂光量子 产率降低, 这与我们的荧光量子产率结果相一致(表 1).

通过紫外光电子能谱法(UPS)测量了该两个高分子 材料的最高电子占据分子轨道(HOMO)能级(图 4), 并汇 总于表 1. 图中给出的全谱图包含二次电子截止边和费 米边(注入势垒), 具体位置采用切线外推法获得, 电离 势(IP)值即理解为相对于真空能级的 HOMO 值. 经计 算, PVBT 和 nPVBT 的 HOMO 能级分别为 -5.73 和 $-5.61 \mathrm{eV}$, 二者差约 $0.1 \mathrm{eV}$, 这可能是由位阻较小的直 链侧链使分子链间距的堆积更紧密所导致 ${ }^{[47]}$. HOMO 能 级减去光学带隙得到最低电子未占分子轨道(LUMO)能 级, PVBT 和 nPVBT 的 LUMO 能级分别为 -3.37 和 $-3.32 \mathrm{eV}$. 相较于 PPV 类高分子材料 MEH-PPV 的 HOMO $(-5.07 \mathrm{eV})$ 和 LUMO $(-2.9 \mathrm{eV})$ 能级, PVBT 和 nPVBT 的能级降低, 是由于 $\mathrm{BT}$ 的强吸电子作用对分子 能级的调节 ${ }^{[43,49]}$.

\section{4 场效应性能的测试}

为了研究高分子材料的电荷传输性质, 我们制备了 基于 PVBT 和 nPVBT 薄膜的顶栅-底接触型 OFET 器件, 并研究了不同温度热处理条件下 $\left(150 、 180 、 210{ }^{\circ} \mathrm{C}\right.$ 热 退火)的器件性能, 结果显示 $180{ }^{\circ} \mathrm{C}$ 热退火 $10 \mathrm{~min}$ 的器 件性能较好 $\left(150{ }^{\circ} \mathrm{C}\right.$ 和 $210{ }^{\circ} \mathrm{C}$ 的器件性能见图 $\mathrm{S} 14$ 及表 S2), 相关的性能数据汇总于表 3. 图 5 给出了所构筑晶 体管器件代表性的转移[图 5(A), 5(C)] 和输出[图 5(B),
5(D)]曲线. PVBT 和 nPVBT 均表现为典型的 $p$ 型电荷传 输特性, 具有理想的栅压调控特性. 从表 3 可以看出, 两个高分子材料的迁移率差距不大, 平均迁移率为 $0.4 \times 10^{-4} \mathrm{~cm}^{2} \cdot \mathrm{V}^{-1} \cdot \mathrm{s}^{-1}$ 和 $0.5 \times 10^{-4} \mathrm{~cm}^{2} \cdot \mathrm{V}^{-1} \cdot \mathrm{s}^{-1}$, 最高迁 移率为 $1.1 \times 10^{-4} \mathrm{~cm}^{2} \cdot \mathrm{V}^{-1} \cdot \mathrm{s}^{-1}$, 器件均展现了较大的开 关比特性, 在 $10^{3} \sim 10^{4}$ 之间. 研究表明, 烷氧基链对迁 移率的影响不大, 图 5(A)和 5(C) 显示电流开平方值 $\left(-I_{\mathrm{DS}}\right)^{1 / 2}$ 与 $V_{\mathrm{G}}$ 的关系曲线接近理想线型, 说明根据饱和 区计算得到的迁移率是可信的 ${ }^{[50]}$. 由于两个高分子材 料较低的 HOMO 能级导致活性层与源电极间的空穴注 入势垒较大, 导致器件展示了较大的阈值电压 $\left(V_{\mathrm{T}}\right)$. 后 续又对 PVBT 和 nPVBT 的晶体管器件测试了回滞曲线 (图 S15), 实验结果可以看出两个材料的器件在工作过 程中回滞较小, 说明活性层界面的缺陷小, 器件具有良 好的电学稳定性.

为了研究膜形态对器件性能的影响, 我们采用掠入 射 X 射线衍射(GIXRD)和原子力显微镜(AFM)对高分子 薄膜的微观结构进行了表征. 图 S16, S17 显示了 PVBT 和 nPVBT 在硅片上退火前后的二维 GIXRD 衍射模式. 数据显示在室温条件下退火的薄膜在 $q_{x y}$ 和 $q_{z}$ 方向衍射 峰很弱或者基本没有衍射峰, 说明室温退火得到的薄膜 基本是无定型态的. 当退火温度升至 $150 、 180 、 210{ }^{\circ} \mathrm{C}$ 时, PVBT 和 nPVBT 的制备薄膜, 均显示出只有一个强 度相当的平面外衍射峰 $\left(q_{z}=0.4 \AA^{-1}\right)$, 即(100)面, 衍射 环由烷氧基侧链的堆积导致, 面内 $q_{x y}$ 方向衍射峰较弱, 说明退火后的薄膜相对退火前有了一定的取向性，但三 个退火温度下的薄膜堆积基本没有差异 ${ }^{[51]}$. 同时又对 薄膜表面形貌进行了测试, 图 6 显示了 $180{ }^{\circ} \mathrm{C}$ 退火 10 $\min$ 条件下制备的 PVBT [图 6(A)] 和 nPVBT [图 6(B)] 薄 膜的 AFM 高度图, 二者薄膜表面表现出连续而均匀的 

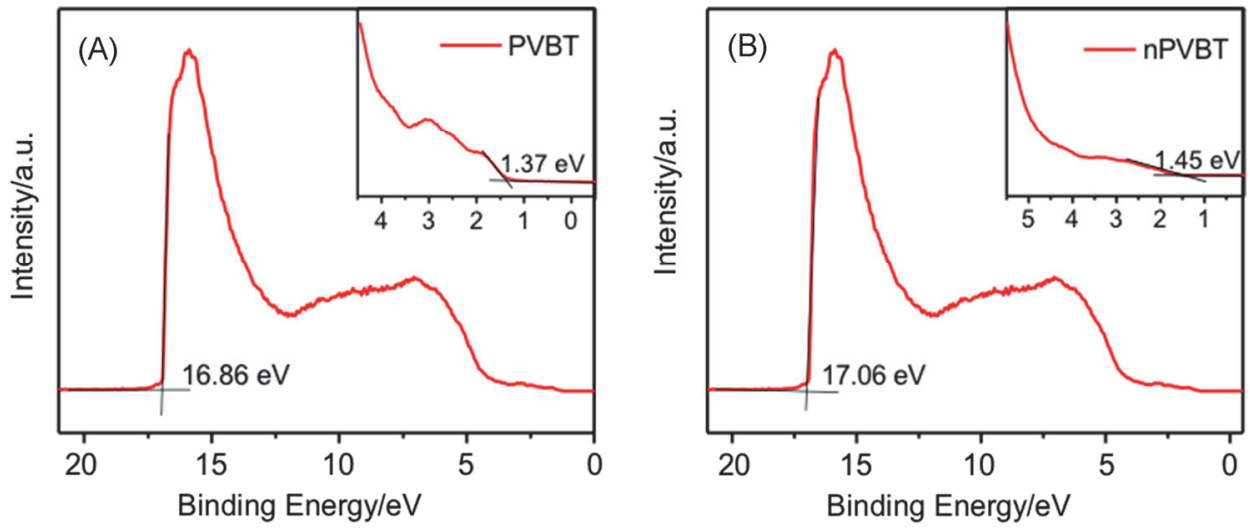

图 4 材料 PVBT (A) 和 nPVBT (B) 的紫外光电子能谱

Figure 4 UPS spectra of PVBT (A) and nPVBT (B)

表 3 PVBT 和 nPVBT 的薄膜器件性能

Table 3 Thin film device characteristics of PVBT and nPVBT

\begin{tabular}{|c|c|c|c|c|c|}
\hline Sample & $T_{\text {annealing }} /{ }^{\circ} \mathrm{C}$ & $\mu_{\text {ave }}{ }^{a} /\left(\mathrm{cm}^{2} \cdot \mathrm{V}^{-1} \cdot \mathrm{s}^{-1}\right)$ & $\mu_{\max }^{b} /\left(\mathrm{cm}^{2} \cdot \mathrm{V}^{-1} \cdot \mathrm{s}^{-1}\right)$ & $V_{\mathrm{T}}^{c} / \mathrm{V}$ & $I_{\mathrm{on} / \mathrm{off}}$ \\
\hline PVBT & 180 & $0.5 \times 10^{-4}$ & $1.1 \times 10^{-4}$ & $30 \sim 40$ & $10^{3} \sim 10^{4}$ \\
\hline nPVBT & 180 & $0.4 \times 10^{-4}$ & $1.1 \times 10^{-4}$ & $30 \sim 40$ & $10^{3} \sim 10^{4}$ \\
\hline
\end{tabular}

${ }^{a}$ Average mobilities measured under ambient conditions; ${ }^{b}$ Maximum mobilities measured under ambient conditions; ${ }^{c}$ Corresponding values of threshold voltage.
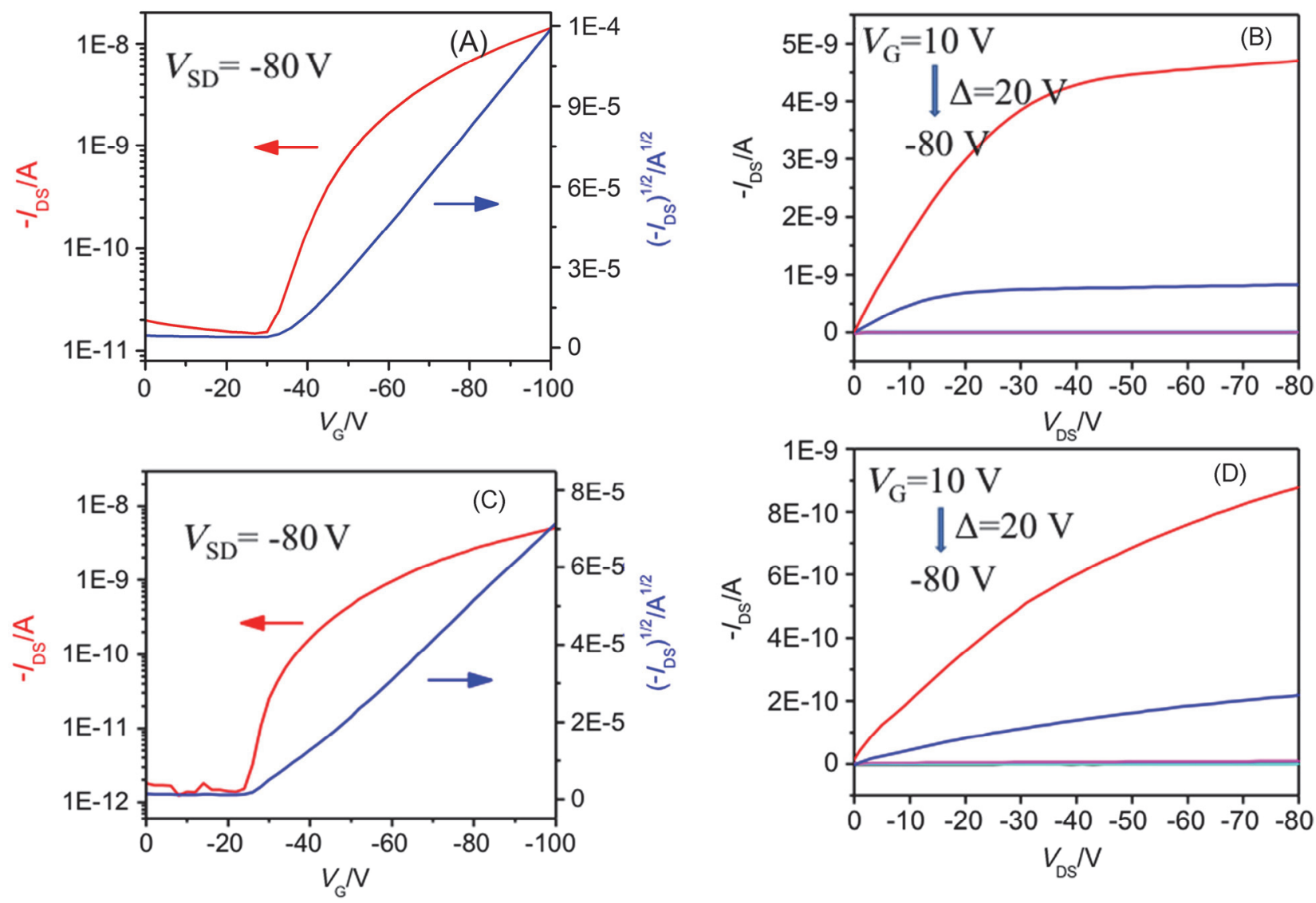

图 5 PVBT $(\mathrm{A}, \mathrm{C})$ 和 $\mathrm{nPVBT}(\mathrm{B}, \mathrm{D})$ 的转移和输出曲线

Figure 5 Typical transfer and output characteristics of PVBT (A, C) and nPVBT (B, D) based field-effect transistors 

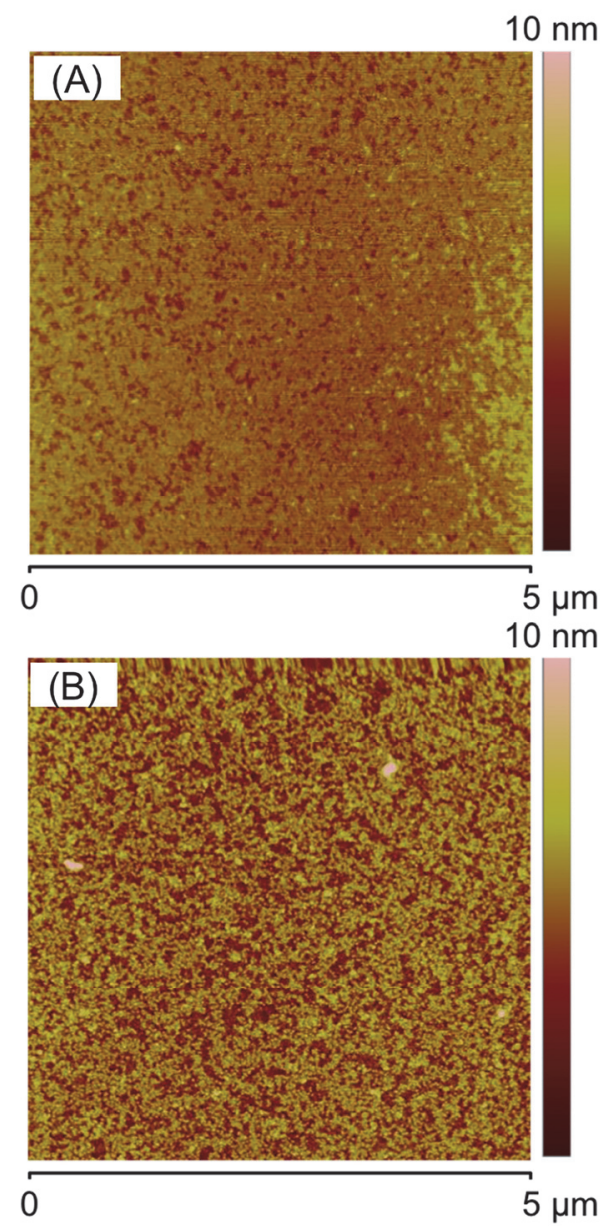

图 6 PVBT (A)和 nPVBT 薄膜(B)的 $\mathrm{AFM}$ 高度图(在 $180{ }^{\circ} \mathrm{C}$ 退火 10 $\min )$

Figure 6 AFM height images of PVBT films (A) and nPVBT films (B) (Films annealed at $180{ }^{\circ} \mathrm{C}$ for $10 \mathrm{~min}$ )

形态，具有较小的聚集结构特征和平滑的粗糙度. PVBT 均方粗粘度约 $0.5 \mathrm{~nm}, \mathrm{nPVBT}$ 均方粗粘度约 $1.0 \mathrm{~nm}$, 没 有形成明显的结晶或纤维状趋势. GIXRD 和 AFM 实验 结果表明两个材料的薄膜经热退火后薄膜结晶性不强, 是一类具有无定形结构特征的新型光电集成共轭高分 子材料.

\section{3 结论}

具有场效应性能高发光有机高分子半导体材料的 发展对于推动有机发光场效应晶体管及其相关器件研 究具有至关重要的意义. 从平衡材料电荷传输和发光性 能的角度出发, 我们提出了基于强发光基元和共轭结构 基元共聚的场效应发光共轭高分子材料的设计思想, 希 望结合侧链工程调控分子间距保持发光基元的强发光 性能的同时, 充分发挥共轭高分子材料中高效链内传输 和链间协同的电荷传输特性, 实现同一材料中光电性能 的集成. 基于该思想的指导, 本工作中我们合成了基于 经典强发光基元苯乙烯和共轭结构基元 BT 的两例新型 共轭高分子材料 PVBT 和 nPVBT, 系统全面地研究了两
个高分子材料的光物理特性和场效应性能. 研究结果表 明, 相比于单一结构基元的 PPV 衍生物材料, 该两例共 聚物材料表现了亮橙色发光特性, 同时由于共轭骨架结 构共轭性的提高和链间紧密堆积, 均展现了高效的电荷 传输特性, 空穴载流子迁移率可达 $1.1 \times 10^{-4} \mathrm{~cm}^{2} \cdot \mathrm{V}^{-1}$. $\mathrm{s}^{-1}$, 是一类新型的光电集成共轭高分子材料, 在 OLET 及相关光电功能器件中具有一定的潜在应用. 这一研究 工作证实了我们对于光电集成共轭高分子材料的设计 思路，同时为其他更高性能共轭高分子光电材料的设计 合成提供了实验依据.

\section{4 实验部分}

\section{1 试剂与仪器}

对苯二酚、氢化钠、澳素、反-1,2-二(三正丁基锡基) 乙烯、 $\mathrm{Pd}\left(\mathrm{PPh}_{3}\right)_{4} 、 4,7$-苯并噻二唑频哪醇硼酸酯等试剂 直接从 Inochem、J\&K、TCI 等试剂公司购买, 未经进一 步纯化. 甲苯与二苯甲酮和金属钠在氩气氛围下回流至 深蓝色后蒸馏使用.

化合物的 ${ }^{1} \mathrm{H}$ 和 ${ }^{13} \mathrm{C}$ 核磁共振谱图均使用 Bruker 400 $\mathrm{MHz}$ 在常温下测得, 溶剂和内标分别为氞代氯仿和四 甲基硅烷. 元素分析用 Flash EA 1112 元素分析仪进行 测定. 质谱是通过高分辨基质辅助激光解析串联离子回 旋共振质谱(HR-MALDI-FT-ICR)由中国科学院化学研 究所分析中心测试所得. 高分子的分子量和分子量分布 (PDI)由 Agilent G7820A PL-GPC220 型凝胶渗透色谱仪 $(\mathrm{GPC})$ 测得, 淋洗剂为 $1,2-$ 二氯苯 $(o-\mathrm{DCB})$, 柱温为 $140{ }^{\circ} \mathrm{C}$. 高分子的热失重分析 (TGA) 是在氮气氛围下, 以 $10 \mathrm{~K} \cdot \mathrm{min}^{-1}$ 的升温速率, 由 STA 409 PC 测试仪测得. 示差扫描量热(DSC) 分析在 Q2000 (TA Instruments, USA)测得, 氮气氛下进行, 升降温速率均为 $10 \mathrm{~K}$ ・ $\mathrm{min}^{-1}$. 紫外-可见吸收光谱(UV-vis)采用 Jasco V-570 光 谱仪测量, 光学带隙由公式 $E_{\mathrm{g}}{ }^{\mathrm{opt}}=1240 / \lambda_{\mathrm{abs}}{ }^{\text {on }}$ 计算获得. 苂光光谱(PL)由 Jasco FP-6600 光谱仪测量. 紫外光电子 能谱(UPS)是在裸硅片上旋涂高分子薄膜由中国科学院 化学研究所分析中心测试所得. 掠入式 $\mathrm{X}$ 射线衍射 (GIXRD) 由中科院高能物理研究所北京同步辐射光源 测得. 薄膜表面形貌及厚度由 Veeco Nanoscope IIId 型 原子力显微镜(AFM)测得.

\section{2 单体和高分子材料的合成}

中间体 1,4-二辛氧基苯 $(\mathbf{1 a}, \mathbf{1 b})$ 和 1,4-二溴-2,5-二辛 氧基苯 $(\mathbf{2 a}, \mathbf{2 b})$ 的合成参见支持信息.

(E)-1,2-双(4-澳-2,5-双(辛氧基)苯基)乙烯(3a, 3b)的 合成: 向 $100 \mathrm{~mL}$ Schlenk 反应管中依次加入反-1,2-二 (三正丁基锡基)乙烯(162.0 mg, $0.266 \mathrm{mmol}$ )、底物 2a (或 者 2b) (654 mg, $1.33 \mathrm{mmol}) 、 \mathrm{Pd}\left(\mathrm{PPh}_{3}\right)_{4}(14.2 \mathrm{mg}, 5 \mathrm{~mol} \%)$ 和甲苯 $(15 \mathrm{~mL})$. 用氩气置换 3 次后, 加热至 $110{ }^{\circ} \mathrm{C}$, 反 应 $3 \mathrm{~h}$ 后即停止加热. 反应液冷却至室温, 得到的反应 液直接蒸干溶剂, 进行硅胶柱层析分离(纯石油醚为淋 
洗剂), 最后得到浅黄色固体 3a (91 mg, 40\%). ${ }^{1} \mathrm{H}$ NMR (400 MHz, Chloroform- $d$ ) $\delta: 7.38(\mathrm{~s}, 2 \mathrm{H}), 7.14(\mathrm{~s}, 2 \mathrm{H})$, $7.08(\mathrm{~s}, 2 \mathrm{H}), 3.90 \sim 3.84(\mathrm{~m}, 8 \mathrm{H}), 1.79 \sim 1.75(\mathrm{~m}, 4 \mathrm{H})$, $1.59 \sim 1.26(\mathrm{~m}, 32 \mathrm{H}), 0.94$ (dt, $J=22.3,7.2 \mathrm{~Hz}, 24 \mathrm{H}) .{ }^{13} \mathrm{C}$ NMR (101 MHz, Chloroform- $d$ ) $\delta: 151.03,149.78,126.93$, $123.75,117.78,111.73,70.22,69.53,31.85,31.83,29.37$, 29.34, 29.32, 29.27, 26.14, 26.06, 22.68, 22.66, 14.12, 14.09. HRMS (MALDI-FT-ICR) $\mathrm{C}_{46} \mathrm{H}_{74} \mathrm{Br}_{2} \mathrm{O}_{4}^{+}$([M] $]^{+}$计算 值为 848.394836 , 实验值为 848.395254. 浅黄色固体 $3 \mathbf{b}$ (113 mg, 45\%): ${ }^{1} \mathrm{H}$ NMR (400 MHz, Chloroform- $d$ ) $\delta$ : $7.35(\mathrm{~s}, 2 \mathrm{H}), 7.11(\mathrm{~s}, 2 \mathrm{H}), 7.07(\mathrm{~s}, 2 \mathrm{H}), 4.02(\mathrm{t}, J=6.5 \mathrm{~Hz}$, $4 \mathrm{H}), 3.95(\mathrm{t}, J=6.6 \mathrm{~Hz}, 4 \mathrm{H}), 1.85 \sim 1.80(\mathrm{~m}, 8 \mathrm{H}), 1.53 \sim$ $1.27(\mathrm{~m}, 40 \mathrm{H}), 0.90 \sim 0.85(\mathrm{~m}, 12 \mathrm{H}) .{ }^{13} \mathrm{C}$ NMR $(101 \mathrm{MHz}$, Chloroform-d) $\delta: 151.04,149.80,126.95,123.76,117.79$, $111.81,111.75,70.23,69.54,31.84,29.37,29.32,29.27$, $26.15,26.06,22.68,22.66,14.12,14.10$. HRMS (MALDI-FT-ICR) $\mathrm{C}_{46} \mathrm{H}_{74} \mathrm{Br}_{2} \mathrm{O}_{4}{ }^{+}\left([\mathrm{M}]^{+}\right)$计算值为 848.394836, 实验值为 848.395359 .

聚(1,2-双(2,5-双(异辛氧基)亚苯基亚乙烯基-2,1,3苯并噻二唑)) (PVBT)和聚(1,2-双(2,5-双(正辛氧基)亚苯 基亚乙烯基-2,1,3-苯并噻二唑)) (nPVBT)的合成: 向 10 $\mathrm{mL}$ Schlenk 反应管中依次加入单体 3a (或者 3b) (127 $\mathrm{mg}, 0.15 \mathrm{mmol}$ )、4,7-苯并噻二唑频哪醇硼酸酯 $(58 \mathrm{mg}$, $0.15 \mathrm{mmol}) 、 \mathrm{Pd}\left(\mathrm{PPh}_{3}\right)_{4}(8 \mathrm{mg}, 5 \mathrm{~mol} \%)$ 、相转移催化剂 Aliquait336、甲苯 $(2 \mathrm{~mL}) 、 \mathrm{~K}_{2} \mathrm{CO}_{3}$ 水溶液 $\left(2 \mathrm{~mol} \cdot \mathrm{L}^{-1}, 0.5\right.$ $\mathrm{mL}$ ). 用氩气置换 3 次后, 加热至 $110{ }^{\circ} \mathrm{C}$, 反应 $24 \mathrm{~h}$. 之 后向反应液加入 1 -溴苯 $(0.5 \mathrm{~mL})$, 继续反应 $12 \mathrm{~h}$. 冷却 至室温, 将反应的甲苯溶液在甲醇中沉降, 经过滤收集 粗聚物, 并通过用甲醇和丙酮的索氏提取法纯化, 将剩 余的产物用热氯仿溶解. 最后通过蒸发浓缩氯仿溶液, 再次沉降到甲醇中, 经抽滤及真空干燥得到最终产物 PVBT $(110 \mathrm{mg})$. GPC $\left(o-\mathrm{DCB}, 140{ }^{\circ} \mathrm{C}\right): M_{\mathrm{n}}=32.5 \mathrm{kDa}$, $M_{\mathrm{w}}=77.4 \mathrm{kDa}, P D I=2.38$; 元素分析: $\mathrm{C}_{52} \mathrm{H}_{78} \mathrm{~N}_{2} \mathrm{O}_{4} \mathrm{~S}$ 计算 值: C, 75.38; H, 9.18; N, 3.42. 实验值: C, 75.50; H, 9.50; N, 3.39. nPVBT $(98 \mathrm{mg}):$ GPC $\left(o-\mathrm{DCB}, 140{ }^{\circ} \mathrm{C}\right): M_{\mathrm{n}}=$ $16.6 \mathrm{kDa}, M_{\mathrm{w}}=27.5 \mathrm{kDa}, P D I=1.65$; 元素分析: $\mathrm{C}_{52} \mathrm{H}_{78} \mathrm{~N}_{2} \mathrm{O}_{4} \mathrm{~S}$ 计算值: C, 75.59; H, 9.14; N, 3.15. 实验值: C, 75.50; H, 9.50; N, 3.39 .

\subsection{OFET 器件的制备与测试}

有机场效应晶体管采用顶棚一底接触结构, 以 0.7 $\mathrm{mm}$ 厚的玻璃作为基底, 依次用去离子水、比例约为 $1 / 2$ 的双氧水/浓硫酸(电炉加热, 煮沸)清洗、去离子水、异 丙醇和丙酮各超声约 $10 \mathrm{~min}$, 最后利用氮气快速吹干. 真空蒸镀腔内通过掩膜版蒸镀源、漏金电极, 沟道宽长 比 $(W / L)$ 为 10 . 高分子薄膜通过溶液旋涂法在手套箱中 制备, 溶剂为 $o-\mathrm{DCB}$, 浓度为 $5.0 \mathrm{mg} \cdot \mathrm{mL}^{-1}$, 以 1500 $\mathrm{r} \cdot \mathrm{min}^{-1}$ 的转速旋涂 $60 \mathrm{~s}$, 得到的膜厚约 $20 \mathrm{~nm}$, 高分子 薄膜在手套箱中 $180{ }^{\circ} \mathrm{C}$ 热退火 $10 \mathrm{~min}$ 后, 在其活性层
上旋涂聚甲基丙烯酸甲酯(PMMA)的乙酸丁酯溶液 (60 $\mathrm{mg} \cdot \mathrm{mL}^{-1}$ ), 然后 $90{ }^{\circ} \mathrm{C}$ 退火 $30 \mathrm{~min}$, 所得介电层厚度约 为 $600 \mathrm{~nm}$, 电容约为 $3.7 \mathrm{nF} \cdot \mathrm{cm}^{-2}$. 最后在蒸镀腔内通过 掩膜板蒸镀约 $100 \mathrm{~nm}$ 厚的铝作为栅极, 场效应晶体管 器件的电学特征测试采用 Keithley 4200 SCS 和 Micromanipulator 6150 探针台在空气中进行.

\section{References}

[1] Huang, F.; Bo, Z. S.; Geng, Y. H.; Wang, X. H.; Wang, L. X.; Ma, Y. G.; Hou, J. H.; Hu, W. P.; Pei, J.; Dong, H. L.; Wang, S.; Li, Z.; Shuai, Z. G.; Li, Y. F.; Cao, Y. Acta Polym. Sin. 2019, 50, 988 (in Chinese). (黄飞, 薄志山, 耿延候, 王献红, 王利祥, 马於光, 侯 剑辉, 胡文平, 裴坚, 董焕丽, 王树, 李振, 帅志刚, 李永舫, 曹 镛, 高分子学报, 2019, 50, 988.)

[2] Dong, H. L.; Fu, X. L.; Liu, J.; Wang, Z. R.; Hu, W. P. Adv. Mater. 2013, 25, 6158 .

[3] Xu, Y.; Yao, H. F.; Hou, J. H. Chin. J. Chem. 2019, 37, 207 (in Chinese). (徐业, 姚惠峰, 侯剑辉, 中国化学, 2019, 37, 207.)

[4] Yang, C. Y.; Jin, W. L.; Wang, J.; Ding, Y. F.; Nong, S.; Shi, K.; Lu, Y.; Dai, Y. Z.; Zhuang, F. D.; Lei, T.; Di, C. A.; Zhu, D. B.; Wang, J. Y.; Pei, J. Adv. Mater. 2018, 30, 1802850.

[5] Li, Q. Q.; Li, Z. Acc. Chem. Res. 2020, 53, 962.

[6] Thomas, T. H.; Harkin, D. J.; Gillett, A. J.; Lemaur, V.; Nikolka, M.; Sadhanala, A.; Richter, J. M.; Armitage, J.; Chen, H.; McCulloch, I.; Menke, S. M.; Olivier, Y.; Beljonne, D.; Sirringhaus, H. Nat. Commun. 2019, 10, 2614.

[7] Feng, L. L.; Gu, P. C.; Dong, H. L.; Yao, Y. F.; Hu, W. P. Chin. Sci. Bull. 2015, 60, 2169 (in Chinese). (冯琳琳, 顾鹏程, 董焕丽, 姚奕 帆, 胡文平, 科学通报, 2015, 60, 2169.)

[8] Ren, X. C.; Yang, F. X.; Gao, X.; Cheng, S. S.; Zhang, X. T.; Dong, H. L.; Hu, W. P. Adv. Energy Mater. 2018, 8, 1801003.

[9] Zheng, Z.; Ni, Z. J.; Zhang, X. T.; Zhen, Y. G.; Dong, H. L.; Zhang, J.; Hu, W. P. Sci. China Mater. 2019, 62, 813 (in Chinese). (郑哲, 倪振杰, 张小涛, 甄永刚, 董焕丽, 张锦, 胡文平, 中国科学材 料, 2019, 62, 813.)

[10] Ni, Z. J.; Dong, H. L.; Wang, H. L.; Ding, S.; Zou, Y.; Zhao, Q.; Zhen, Y. G.; Liu, F.; Jiang, L.; Hu, W. P. Adv. Mater. 2018, 30, 1704843.

[11] Ni, Z. J.; Wang, H. L.; Dong, H. L.; Dang, Y. F.; Zhao, Q.; Zhang, X. T.; Hu, W. P. Nat. Chem. 2019, 11, 271.

[12] Guo, Y. L. Acta Polym. Sin. 2020, 51, 448 (in Chinese). (郭云龙, 高 分子学报, 2020, 51, 448.)

[13] Yao, Y. F.; Dong, H. L.; Liu, F.; Russell, T. P.; Hu, W. P. Adv. Mater 2017, 29, 1701251.

[14] Zhao, S.; Zhu, R. Acta Chim. Sinica 2019, 77, 1250 (in Chinese). (赵帅, 朱荣, 化学学报, 2019, 77, 1250.)

[15] Qiu, G. G.; Jiang, Z. Y.; Ni, Z. J.; Wang, H. L.; Dong, H. L.; Zhang, J. Q.; Zhang, X. T.; Shu, Z. B.; Lu, K.; Zhen, Y. G.; Wei, Z. X.; Hu, W. P. J. Mater. Chem. C 2017, 5, 566.

[16] Gu, P. C.; Hu, M. X.; Ding, S.; Zhao, G. Y.; Yao, Y. F.; Liu, F.; Zhang, X. T.; Dong, H. L.; Wang, X. K.; Hu, W. P. Chinese Chem. Lett. 2018, 29, 1675 (in Chinese). (顾鹏程，胡梦笑，丁尚，赵广耀， 姚奕帆，刘峰，张小涛，董焕丽，王祥科，胡文平，中国化学快 报, 2018, 29, 1675.)

[17] Li, C. G.; Wang, Y. S.; Zou, Y.; Zhang, X. T.; Dong, H. L.; Hu, W. P. Angew. Chem. Int. Ed. 2020, 59, 9403.

[18] Mei, J.; Leung, N. L.; Kwok, R. T.; Lam, J. W.; Tang, B. Z. Chem. Rev. 2015, 115, 11718.

[19] Dong, H. L.; Yan, Q. Q.; Hu, W. P. Acta Polym. Sin. 2017, 8, 1246 (in Chinese). (董焕丽, 燕青青, 胡文平, 高分子学报, 2017, 8, 1246.)

[20] Qian, X.; Su, M.; Li, F. Y.; Song, Y. L. Acta Chim. Sinica 2016, 74, 565 (in Chinese). (钱金金, 苏萌, 李风煜, 宋延林, 化学学报, 2016, 74, 565.)

[21] Xu, X. N.; Han, B.; Yu, X.; Zhu, Y. Y. Acta Chim. Sinica 2019, 77, 485 (in Chinese). (许晓娜, 韩宾, 于曦, 朱艳英, 化学学报, 2019, 77, 485.)

[22] Zhang, Y. H.; Ye, J.; Liu, Z. Y.; Liu, Q. Q.; Guo, X. F.; Dang, Y. F.; Zhang, J. Q.; Wei, Z. X.; Wang, Z. X.; Wang, Z. H.; Dong, H. L.; Hu, W. P. J. Mater. Chem. C 2020, DOI: 10.1039/D0TC01174F. 
[23] Fu, Y.; Wang, F.; Zhang, Y.; Fang, X.; Lai, W. Y.; Huang, W. Acta Chim. Sinica 2014, 72, 158 (in Chinese). (付钰, 王芳, 张燕, 方旭, 赖文勇, 黄维, 化学学报, 2014, 72, 158.)

[24] Wang, Z. W.; Guo, S. J.; Li, H. W.; Wang, B.; Sun, Y. T.; Xu, Z. Y.; Chen, X. S.; Wu, K. J.; Zhang, X. T.; Xing, F. F.; Li, L. Q.; Hu, W. P. Adv. Mater. 2019, 31, 1805630.

[25] Hepp, A.; Heil, H.; Weise, W.; Ahles, M.; Schmechel, R.; Seggern, H. V. Phys. Rev. Lett. 2003, 91, 157406.

[26] Zhang, C. C.; Chen, P. L.; Hu, W. P. Small 2016, 12, 1252.

[27] Muhieddine, K.; Ullah, M.; Pal, B. N.; Burn, P.; Namdas, E. B. Adv. Mater. 2014, 26, 6410.

[28] Qin, Z. S.; Gao, H. K.; Liu, J. Y.; Zhou, K.; Li, J.; Dang, Y. Y.; Huang, L.; Deng, H. X.; Zhang, X. T.; Dong, H. L.; Hu, W. P. Adv. Mater. 2019, 31, 1903175.

[29] Liu, C. F.; Liu, X.; Lai, W. Y.; Huang, W. Adv. Mater. 2018, 30, 1802466.

[30] Ma, Y. G.; Shen, J. C. Sci. Sin. Chim. 2007, 37, 105 (in Chinese). (马 於光, 沈家骢, 中国科学, 2007, 37, 105.)

[31] Zhang, X. T.; Dong, H. L.; Hu, W. P. Adv. Mater. 2018, 30, 1801048.

[32] Xie, Z. Y.; Liu, D.; Zhang, Y. H.; Liu, Q. Q.; Dong, H. L.; Hu, W. P. Chem. J. Chin. Univ. 2020, 41, 1179 (in Chinese). (谢子仪, 刘单, 张逸寒, 刘情情, 董焕丽, 胡文平, 高等学校化学学报, 2020, 41, 1179.)

[33] Liu, D.; De, J. B.; Gao, H. K.; Ma, S. Q.; Ou, Q.; Li, S.; Qin, Z. S.; Dong, H. L.; Liao, Q.; Xu, B.; Peng, Q.; Shuai, Z. G.; Tian, W. J.; Fu, H. B.; Zhang, X. T.; Zhen, Y. G.; Hu, W. P. J. Am. Chem. Soc. 2020, 142, 6332 .

[34] Liu, H. C.; Yao, L.; Li, B.; Chen, X. K.; Gao, Y.; Zhang, S. T.; Li, W. J.; Lu, P.; Yang, B.; Ma, Y. G. Chem. Commun. 2016, 52, 7356.

[35] Chen, M. Y.; Zhao, Y.; Yan, L. J.; Yang, S.; Zhu, Y. N.; Murtaza, I.; He, G. F.; Meng, H.; Huang, W. Angew. Chem. Int. Ed. 2016, 128, 1.
[36] Chen, Z. K.; Lee, N. H. S.; Huang, W. Macromolecules 2003, 36, 1009.

[37] Burroughes, J. H.; Bradley, D. D. C.; Brown, A.; Marks, R. R.N.; Mackay, K.; Friend, R. H.; Burnst, P. L.; Holmest A. B. Nature 1990, 347, 539

[38] Yu, G.; Gao, J.; Hummelen, J. C.; Wudl, F.; Heeger, A. J. Science 1995, 270, 1789.

[39] Tessler, N.; Denton, G. J.; Friend, R. H. Nature 1996, 382, 695.

[40] Zaumseil, J.; Friend, R. H.; Sirringhaus, H. Nat. Mater. 2006, 5, 69.

[41] Gambino, S.; Bansal, A. K.; Samuel, I. D. W. Org. Electron. 2013, 14,1980 .

[42] Johansson, D. M.; Theander, M.; Srdanov, G.; Yu, G.; Inganas, O.; Andersson, M. R. Macromolecules 2001, 34, 3716.

[43] Anant, P.; Lucas, N. T.; Jacob, J. Org. Lett. 2008, 10, 5533.

[44] Zhang, W. M.; Smith, J.; Watkins, S. E.; Gysel, R.; McGehee M.; Salleo, A.; Kirkpatrick, J.; Ashraf, S.; Anthopoulos, T.; Heeney, M.; McCulloch, I. J. Am. Chem. Soc. 2010, 132, 11437.

[45] Wen, S. P.; Pei, J. N.; Zhou, Y. H.; Li, P. F.; X, L. L.; Li, Y. W.; Xu, B.; Tian, W. J. Macromolecules 2009, 42, 4977.

[46] Gwinner, M. C.; Kabra, D.; Roberts, M.; Brenner, T. J. K.; Wallikewitz, B. H.; McNeill, C. R.; Friend, R. H.; Sirringhaus, H. Adv. Mater. 2012, 24, 2728.

[47] Lei, T.; Dou, J. H.; Pei, J. Adv. Mater. 2012, 24, 6457.

[48] Shahid, M.; Ashraf, R. S.; Klemm, E.; Sensfuss, S. Macromolecules 2006, 39, 7844.

[49] Li, Y. F.; Cao, Y.; Gao, J.; Wang, D. L.; Yu, G.; Heeger, A. J. Synthetic Met. 1999, 99, 243.

[50] Sirringhaus, H. Adv. Mater. 2014, 26, 1319.

[51] Rivnay, J. M. S. C.; Miller, C. E.; Salleo, A.; Toney, M. F. Chem. Rev. 2012, 112, 5488. 